\title{
Transmembrane receptor CXCR7 increases the risk of extrahepatic metastasis of relatively well-differentiated hepatocellular carcinoma through upregulation of osteopontin
}

\author{
TONG-CHUN XUE ${ }^{1,2}$, RONG-XIN CHEN ${ }^{1,2}$, ZHENG-GANG REN ${ }^{1,2}$, JING-HUAI ZOU ${ }^{1,2}$, \\ ZHAO-YOU TANG ${ }^{1,2}$ and SHENG-LONG YE ${ }^{1,2}$ \\ ${ }^{1}$ Liver Cancer Institute, Zhongshan Hospital, Fudan University, Shanghai; ${ }^{2}$ Key Laboratory of Carcinogenesis \\ and Cancer Invasion, Fudan University, Ministry of Education, Shanghai, P.R. China
}

Received December 27, 2012; Accepted February 14, 2013

DOI: $10.3892 /$ or.2013.2442

\begin{abstract}
Recurrence and metastasis are the main obstacles to improving the survival of patients with post-resective hepatocellular carcinoma (HCC). Our previous study suggests a critical role of CXCR7 in the metastasis of HCC. In the present study, the effect of CXCR7 as a risk factor for metastasis of HCC was evaluated. Immunohistochemical assay was performed on tissue microarrays based on $\mathrm{HCC}$ with extrahepatic metastases after hepatectomy. Two categories based on staining scores were used to evaluate the risk effect of CXCR7, respectively. The effect of CXCR7 on osteopontin (OPN) was explored by RNA interference. Based on the results, in both categories, highly expressed CXCR7 was a dependent risk factor for extrahepatic metastasis because of the potential association with relatively good cell differentiation. Stratification analyses indicated that CXCR7 was a strong independent risk factor (OR, 3.40; 95\% CI, 1.07-18.84; $\mathrm{P}=0.038$ in category 1 and OR, 6.40; 95\% CI, 1.64-24.92; $\mathrm{P}=0.007$ in category 2 , respectively) in patients with Edmondson grade 1/2. Furthermore, CXCR7 correlated well and positively with expression of OPN ( $\mathrm{P}=0.019$ and $\mathrm{P}<0.001$ in two categories, respectively) in HCC cases with Edmondson grade 1/2. Immunocytochemistry and RT-PCR demonstrated downregulation of OPN in a highly metastatic HCC cell line following knockdown of CXCR7. Taken together, these findings suggest that high expression of CXCR7 increases the risk of metastasis in post-resective HCC patients with relatively good differentiated tumors, potentially through upregulation of OPN. This group of patients may acquire a survival benefit from early detection and treatment of recurrence and metastasis.
\end{abstract}

Correspondence to: Professor Sheng-Long Ye, Liver Cancer Institute, Zhongshan Hospital, Fudan University, 180 Fenglin Road, Shanghai 200032, P.R. China

E-mail: slye@shmu.edu.cn

Key words: CXCR7, hepatocellular carcinoma, differentiation, metastasis, osteopontin

\section{Introduction}

Recurrence and metastasis are still the main obstacles to improving the survival of patients with hepatocellular carcinoma $(1,2)$ and are associated with highly increased morbidity and mortality worldwide. Several risk factors including tumor size, microvessel invasion and poor differentiation, have been demonstrated to increase the risk of recurrence and even extrahepatic metastasis of post-resective HCC patients (1) and their identification can aid in carrying out more positive treatment strategies, such as adjuvant TACE. However, the heterogeneity and molecular biological characteristics of HCC increase the complexity and difficulties of therapy. Providing treatment according to the differential characteristics of specific subgroups of HCC may decrease the potential of recurrence and metastasis, and this is becoming the future developmental trend in treatment strategy.

Chemokine receptor CXCR7, belonging to one of the G-protein coupled seven-transmembrane receptors, has the definitive ligand stromal cell-derived factor- $1 \alpha($ SDF- $1 \alpha)$ and truncated ITAC (CXCL11) $(3,4)$. Accumulated research has shown chemokine receptors to be pertinent to the progression of tumors (5). The level of CXCR7 expression has been demonstrated to be closely related to the invasive activities of prostate cancer cells (6). In addition, higher expression of CXCR7 is linked to the early and metastatic recurrence in pathological stage I non-small cell lung cancer (7). Recently, overexpression of CXCR7 was found in the HCC cell line SMMC-7721 and tumor tissues (8). Moreover, CXCR7 can also be expressed by endothelial cells (9). Our previous research showed that downregulation of CXCR7 inhibited the metastasis of HCC (10). However, the potential merit of CXCR7 as a risk factor for extrahepatic metastasis is unclear.

In the present study, we evaluated the predictive role of CXCR7 as a risk factor in metastasis of post-resective human HCC. According to the immunohistochemical staining of the tissue microarray, high expression of CXCR7 increased the risk of extrahepatic metastasis prominently as an independent risk factor based on cell differentiation. Furthermore, the close relationship between CXCR7 and osteopontin (OPN) was found and was confirmed by immunocytochemistry and 
RT-PCR analysis of OPN after downregulation of CXCR7 in a highly metastatic HCC cell line by RNA interference (RNAi).

\section{Materials and methods}

Cell line. The highly metastatic human HCC cell line HCCLM-3 (100\% lung metastatic potential) was used, which was established at the Liver Cancer Institute of Fudan University (Shanghai, China). The cell line was cultured in high glucose DMEM (Gibco-BRL, Grand Island, NY, USA) supplemented with $10 \%$ fetal bovine serum (HyClone Laboratories, Inc., Logan, UT, USA).

Patients. One hundred and sixteen patients were retrieved from the prospectively designed database. Ethical approval was obtained from the Zhongshan Hospital Research Ethics Committee, and informed consent was obtained from each patient. The patients underwent hepatectomy by the same surgical team from January 2000 to May 2004. The hepatectomy of HCC was carried out as previously described (11). The patients were pathologically confirmed as having HCC. Paraffin tissue sections were stained by hematoxylin and eosin, and reviewed by two pathologists according to the WHO histomorphologic criteria. There were 103 male and 13 female patients with a mean age of $51.0 \pm 11.4$ years (range, 18-78 years). Ninety-four patients were positive for the hepatitis B surface antigen (HBsAg). All patients were classified as Child-Pugh A.

Follow-up. Regular follow-up procedures in our clinic are as follows: serum $\alpha$ fetoprotein (AFP) assay and liver ultrasonography every 3 months during the first year, then every 6 months; and magnetic resonance imaging (MRI) or computed tomography (CT) scanning after 1 month, then every 6 months. Chest CT scanning was regularly used to examine the lung metastasis. Lung metastasis was confirmed by biopsy through endoscopy or pathology after partial pulmonary resection. Until May 2009, 58 patients were found to present with lung metastasis. Ten patients with resectable lung metastasis received partial resection of the lung.

TMA and immunohistochemistry. Hematoxylin and eosinstained slides were screened for optimal tumor content and tissue adjacent to tumor (TAT) with a distance of $2 \mathrm{~cm}$. The TMA was then constructed in accordance with standard procedures (12) based on 116 tumor tissues and 47 TATs. Two cores were taken from each formalin-fixed, paraffin-embedded HCC samples by using punch cores that measured $1.0 \mathrm{~mm}$ in diameter from the center of tumor foci and TAT. A two-step method of immunohistochemistry including heat-induced antigen-retrieval procedure was performed in a standard manner. Rabbit anti-human CXCR7 IgG (Novus Biologicals, Littleton, CO, USA) at 1:200 and rabbit anti-human osteopontin IgG (Santa Cruz Biotechnology, Santa Cruz, CA, USA) at 1:200 were used as primary antibodies for detection. Detection without the primary antibody was considered as the negative control.

Scoring and categories of CXCR7 expression. The immunoassay was defined by the staining intensity and the percentage of positive tumor cells as previously described (13). Two pathologists observed the results independently. For analysis of convenience, we simplified the assay results into three expression levels as negative, weak and strong. Based on the three expression levels, two categories for further analysis were set up as follows: category 1 , negative and weak staining were recognized as CXCR $7^{\text {neg+low }}$ and strong staining was recognized as CXCR $7^{\text {high }}$; category 2 , only detectable staining of CXCR7 expression was analyzed. Weak staining was recognized as CXCR $7^{\text {low }}$ and strong staining was recognized as CXCR $7^{\text {high }}$.

$R N A i$. Small interference RNA (siRNA) transfection of HCCLM 3 cells was performed according to the Lipofectamine 2000 protocol (14). After $24 \mathrm{~h}$, immunocytochemistry assay was performed as previously described (15) except that 1:800 rabbit anti-human OPN IgG (Millipore Corp., Billerica, MA, USA) was used as the primary antibody. RT-PCR was also used to determine the mRNA level of OPN: sense, 5'-GGACTCCATTGACTCGAACG-3'; antisense, 5'-TAA TCTGGACTGCTTGTGGC-3'.

Statistical analysis. Pearson's Chi-square test was used to compare qualitative variables in the univariate analysis. When expected sample values were $<5$, Fisher's exact test was used. Twelve potential risk factors including CXCR7 for development of lung metastasis of HCC were all categorized variables. These included 4 clinical factors [age $\leq 60$ years or $>60$ years; gender, HBsAg status and serum AFP level $\leq 20 \mu \mathrm{g} / \mathrm{l}$ or $>20 \mu \mathrm{g} / \mathrm{l}(16)]$, and 8 pathological factors (cirrhosis or non-cirrhosis, tumor size $\leq 5 \mathrm{~cm}$ or $>5 \mathrm{~cm}$, single or multiple tumor nodules, well or poorly encapsulated tumor, the presence or absence of microvascular invasion, portal lymphatic status, Edmondson grade 1/2 [well- and moderately differentiated grades) or grade $3 / 4$ (poorly differentiated grade) and CXCR7 staining]. Potential risk factors were selected from univariate analysis when $\mathrm{P}<0.25$. Binary logistic regression was used as multivariate analysis to evaluate the screened risk factors. Receiver operation curve (ROC) was used further to confirm the predictive accuracy of the risk factors. Spearman's rank test was used to detect the correlation between CXCR7 and OPN. One-way ANOVA was used for intergroup comparisons. All P-values were 2-tailed and the statistical significance was set at 0.05. Statistical analyses were carried out using SPSS 18.0 software (SPSS Inc., Chicago, IL, USA).

\section{Results}

Association between high expression of CXCR7 and extrahepatic metastasis. The immunopositivity for CXCR7 was mainly observed in the membrane and cytoplasm of HCC cells. Typically, the stained HCC cells were mostly round or oval with increased karyoplasm ratio (Fig. 1). Differential staining intensity of CXCR7 expression from negative to strong was observed in 20, 45 and 51 patients, respectively (Fig. 1).

In category $1,51.7 \%$ cases showed CXCR $7^{\text {high }}$ in the patients with lung metastasis $(n=58)$, whereas in the patients without lung metastasis $(\mathrm{n}=58)$, only $36.2 \%$ cases showed $\mathrm{CXCR} 7^{\text {high }}$. Immunopositivity for CXCR7 in patients with lung metastasis was potentially stronger than that in patients without metastasis 


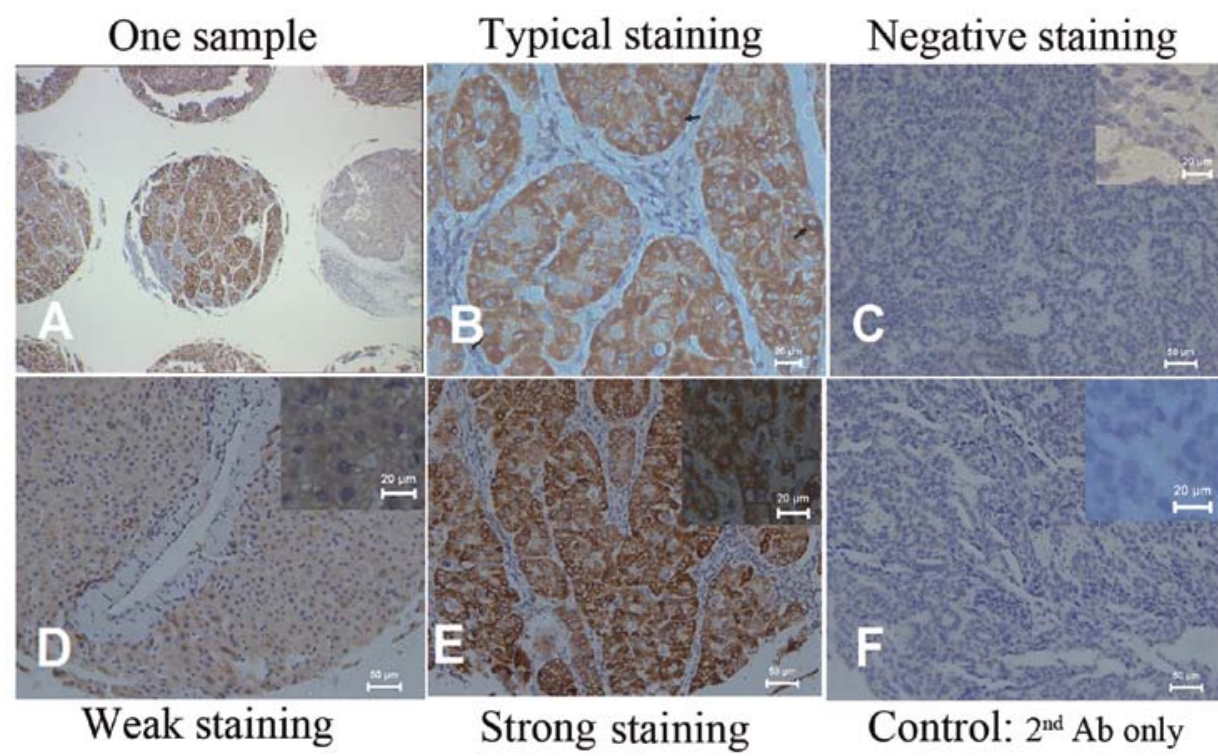

Figure 1. Immunohistochemical detection of CXCR7 in hepatocellular carcinoma (HCC). (A) One sample from a tumor tissue in the TMA. (B) Representative image of immunohistochemical detection of CXCR7 indicates that the positively stained tumor cells were mostly round or oval with increased karyoplasm ratio and localized or scattered staining. Immunopositivity for CXCR7 is indicated by arrows and was mainly observed in the membrane and the cytoplasm of tumor cells typically (original magnification, $\mathrm{x} 400$; scale bar, $20 \mu \mathrm{m}$ ). (C-E) Tumor staining included 3 types: from negative staining to strong staining. (F) Negative control indicates the specificity of the antibodies in tumor tissue. (C-F, original magnification, x200; scale bar, $50 \mu \mathrm{m}$ ). The corresponding x400 original magnification of tumor staining is shown at the top right corner. Scale bar, $20 \mu \mathrm{m}$.

[hazards ratio (HR), 1.89; 95\% confidence interval (CI), 0.90 3.97; $\mathrm{P}=0.092]$. Furthermore, in category 2 , patients with lung metastasis showed significantly strong CXCR7 staining (HR, $2.59 ; 95 \%$ CI, 1.13-5.92; $\mathrm{P}=0.023)$.

In TAT analysis, no statistical difference was noted between patients with lung metastasis $(n=23)$ and those without metastasis $(n=24)$ for CXCR7 staining $(P=0.302$ in category 1). Furthermore, among 23 TATs of HCC with lung metastasis, only microvascular invasion was found to be associated with CXCR7 staining in category 1 . Tumor cells with high expression of CXCR7 in TATs of patients exhibited stronger microvascular invasion (HR, 10.67; 95\% CI, 1.04109.94; $\mathrm{P}=0.027)$.

Role of CXCR7 in the increased risk of extrahepatic metastasis is correlated with cell differentiation. In category 1 , among the 58 patients with lung metastasis, patients with CXCR $7^{\text {high }}$ appeared to have better differentiated tumors (HR, 0.38; 95\% CI, 0.13-1.08; $\mathrm{P}=0.067)$ but increased AFP level (HR, 4.21; 95\% CI, 1.15-14.39; $\mathrm{P}=0.024)$. Univariate analysis indicated that 6 evaluated factors including CXCR7 staining $(\mathrm{P}=0.092)$, AFP level $(\mathrm{P}=0.051)$, microvascular invasion $(\mathrm{P}=0.003)$, Edmondson grade $(\mathrm{P}=0.012)$, portal lymphatic status $(\mathrm{P}=0.061)$ and tumor capsule $(\mathrm{P}=0.126)$ were potential risk factors. Multivariate analysis did not indicate a significant predictive effect for CXCR7 [odds ratio (OR), 1.89; 95\% CI, 0.84-4.25; $\mathrm{P}=0.125]$. However, when CXCR7 staining and AFP were maintained in the model, CXCR7 and AFP had significant effects on the model (OR, 8.446; 95\% CI, 1.2457.45; $\mathrm{P}=0.029)$.

To further confirm the potential relationship between CXCR7 and cell differentiation in category 1 , we excluded the patients for whom CXCR7 expression could not be detected, and who stained negative from the observations in category 2 . In patients with lung metastasis and detectable CXCR7 expression $(n=46)$, Edmondson grade (HR, 0.26; 95\% CI, 0.07-0.96; $\mathrm{P}=0.038$ ) was associated with CXCR7 staining. Among the 96 patients with detectable CXCR7 expression, univariate analysis showed that 6 factors including CXCR7 staining $(\mathrm{P}=0.023)$, AFP level $(\mathrm{P}=0.007)$, microvascular invasion $(\mathrm{P}=0.025)$, Edmondson grade $(\mathrm{P}=0.015)$, portal lymphatic status $(\mathrm{P}=0.052)$ and tumor capsule $(\mathrm{P}=0.114)$ were potential risk factors. Multivariate analysis showed that microvascular invasion (OR, 3.28; 95\% CI, 1.23-8.78; $\mathrm{P}=0.018)$, Edmondson grade (OR, 3.87; 95\% CI, 1.46-10.24; $\mathrm{P}=0.006)$, and AFP (OR, 3.54; 95\% CI, 1.35-9.28; $\mathrm{P}=0.010)$ were 3 independent risk factors for lung metastasis. When CXCR7 staining was maintained in the model, CXCR7 staining (OR, 4.73; 95\% CI, 1.36-16.48; $\mathrm{P}=0.015$ ) was a dependent risk factor due to potential interaction of CXCR7 expression and Edmondson grade (OR, 0.14; 95\% CI, 0.02-1.02; $\mathrm{P}=0.053$ ). HCC patients with $\mathrm{CXCR} 7^{\text {high }}$ had nearly a 5-fold higher risk to develop lung metastasis than patients with $\mathrm{CXCR} 7^{\text {low }}$.

Stratification analyses of the independent risk role of CXCR7 based on cell differentiation. Due to the potential relationship between highly expressed CXCR7 and relatively good differentiation of tumor cells, we further analyzed the risk predictive role of CXCR7 in patients with Edmondson grade $1 / 2(n=73)$. None of the clinicopathological factors were associated with the expression of CXCR7 staining in category 1 (data not shown). In univariate analysis, 6 factors including CXCR7 staining $(\mathrm{P}=0.017)$, AFP level $(\mathrm{P}=0.003)$, microvascular invasion $(\mathrm{P}=0.008)$, number of tumor nodules $(\mathrm{P}=0.044)$, portal lymphatic status $(\mathrm{P}=0.025)$ and tumor capsule $(\mathrm{P}=0.134)$ were potential risk factors. Multivariate analysis showed that CXCR7 staining (OR, 3.4; 95\% CI, 1.0710.84; $\mathrm{P}=0.038)$, microvascular invasion $(\mathrm{OR}, 4.07 ; 95 \% \mathrm{CI}$, 
$\mathbf{A}$

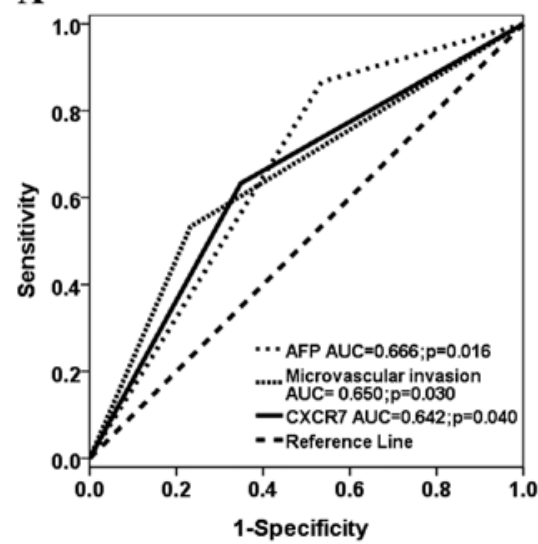

B

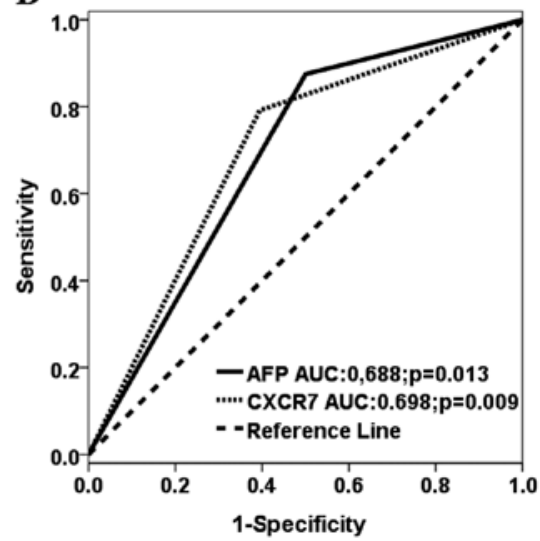

Figure 2. Receiver operation curve (ROC) analysis of CXCR7 and AFP in stratification analysis based on relatively good cellular differentiation. (A) Areas under the curve (AUC) of CXCR7, AFP, and microvascular invasion in category 1 were $>0.05(\mathrm{P}<0.05)$. (B) AUC of CXCR7 and AFP were $>0.05$ ( $\mathrm{P}<0.05)$.

A

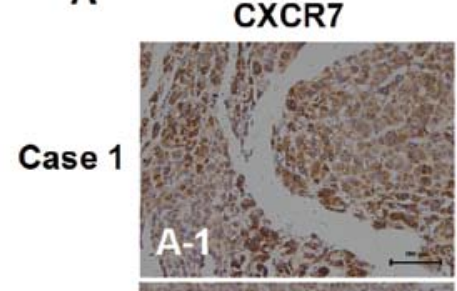

Case 2

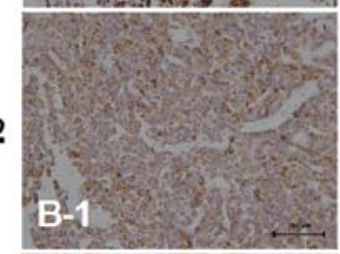

Case 3

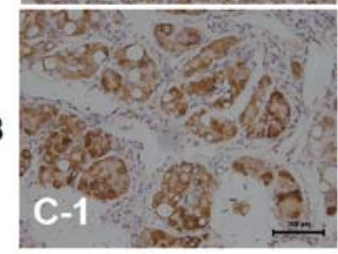

OPN
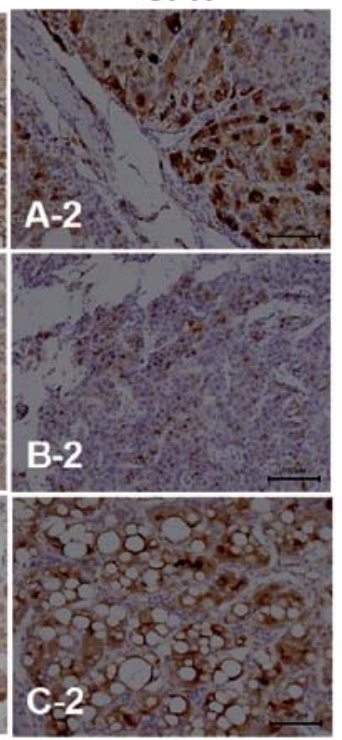

B

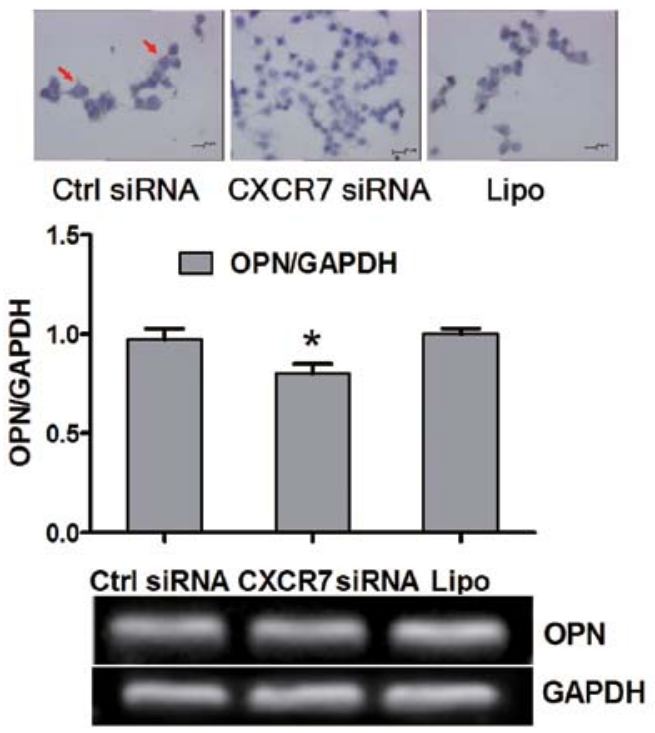

Figure 3. Association between CXCR7 and OPN in HCC. (A) Immunohistochemical staining of CXCR7 and OPN in three representative cases with relatively good differentiation. Analyses indicate the strong consistency in the distribution and staining intensity between the two proteins. (B) Suppression of OPN expression by targeting CXCR7. HCCLM3 cells were treated with CXCR7 siRNA, control siRNA (Ctrl siRNA), or Lipofectamine 2000 (Lipo) before assays. OPN expression was detected by immunocytochemistry (top) and RT-PCR (bottom). (OPN antibody, 1:800). Red arrow indicates the OPN expression in tumor cells. "P<0.01 vs. control siRNA group and Lipofectamine 2000 group.

$1.23-13.48 ; \mathrm{P}=0.021)$ and $\mathrm{AFP}(\mathrm{OR}, 8.18 ; 95 \% \mathrm{CI}, 1.81-36.92$ $\mathrm{P}=0.006)$ were 3 independent risk factors for lung metastasis of patients with well and moderately differentiated HCC. HCC patients with $\mathrm{CXCR} 7^{\text {high }}$ had a 3.4-fold higher risk for developing lung metastasis than patients with CXCR7 $7^{\text {neg+low }}$ In the ROC analysis, three factors predicted the development of lung metastasis, with AUC of 0.642 (95\% CI, 0.512-0.772; $\mathrm{P}=0.040)$ for CXCR7 staining; AUC of 0.666 (95\% CI, 0.541-0.790; $\mathrm{P}=0.016)$ for AFP and AUC of 0.650 (95\% CI, 0.519-0.781; $\mathrm{P}=0.030$ ) for microvascular invasion (Fig. 2A) In addition, we also analyzed the potential risk factors in 43 patients with poorly differentiated tumors (Edmondson grade 3/4). Univariate analysis showed that no potential risk factor was associated with lung metastasis except for microvascular invasion $(\mathrm{P}=0.065)$.
In category 2, stratification analysis of cell differentiation, or Edmondson grade 1/2 $(n=62)$ further confirmed the risk predictive role of $\mathrm{CXCR} 7$ in category 1 . In univariate analysis, 6 factors including CXCR7 staining $(\mathrm{P}=0.002)$ were potential risk factors (Table I). Multivariate analysis showed that CXCR7 staining (OR, 6.40; 95\% CI, 1.64-24.92; $\mathrm{P}=0.007)$ and AFP (OR, 9.32; 95\% CI, 1.76-49.36; $\mathrm{P}=0.009)$ were 2 independent risk factors for lung metastasis of patients with Edmondson grade 1/2. Patients with $\mathrm{CXCR}^{\text {high }}$ had a 6.4 -fold higher risk of developing lung metastasis than patients with CXCR $7^{\text {low }}$ based on the differentiation of the tumor cells. In the ROC analysis, both factors accurately predicted the occurrence of lung metastasis, with AUC of 0.698 (95\% CI, 0.565-0.832; $\mathrm{P}=0.009)$ for CXCR7 staining and AUC of 0.688 (95\% CI, 0.555-0.820; P=0.013) for AFP (Fig. 2B). 
Table I. Univariate analysis of risk factors for metastasis in category 2 based on Edmondson grade 1/2.

\begin{tabular}{|c|c|c|c|}
\hline \multirow[b]{2}{*}{ Variable } & \multicolumn{2}{|c|}{ No. of patients } & \multirow[b]{2}{*}{$\mathrm{P}$-value } \\
\hline & $\begin{array}{l}\text { With lung } \\
\text { metastasis } \\
(n=24)\end{array}$ & $\begin{array}{l}\text { Without lung } \\
\text { metastasis } \\
(n=38)\end{array}$ & \\
\hline \multicolumn{4}{|l|}{ Age (years) } \\
\hline$\leq 60$ & 17 & 30 & 0.467 \\
\hline$>60$ & 7 & 8 & \\
\hline \multicolumn{4}{|l|}{ Gender $^{\mathrm{a}}$} \\
\hline Male & 23 & 33 & 0.391 \\
\hline Female & 1 & 5 & \\
\hline \multicolumn{4}{|l|}{$\mathrm{HBsAg}$} \\
\hline Negative & 6 & 5 & 0.234 \\
\hline Positive & 18 & 33 & \\
\hline \multicolumn{4}{|l|}{ Cirrhosis } \\
\hline Absent & 20 & 27 & 0.271 \\
\hline Present & 4 & 11 & \\
\hline \multicolumn{4}{|l|}{$\operatorname{AFP}(\mu \mathrm{g} / 1)$} \\
\hline$\leq 20$ & 3 & 19 & 0.003 \\
\hline$>20$ & 21 & 19 & \\
\hline \multicolumn{4}{|l|}{ Tumor size (cm) } \\
\hline$\leq 5$ & 6 & 15 & 0.241 \\
\hline$>5$ & 18 & 23 & \\
\hline \multicolumn{4}{|l|}{ No. of tumor nodules } \\
\hline Single & 14 & 31 & 0.046 \\
\hline Multiple & 10 & 7 & \\
\hline \multicolumn{4}{|l|}{ Tumor capsule } \\
\hline Well capsulated & 12 & 26 & 0.147 \\
\hline Poorly capsulated & 12 & 12 & \\
\hline \multicolumn{4}{|c|}{ Microvascular invasion } \\
\hline Negative & 12 & 28 & 0.058 \\
\hline Positive & 12 & 10 & \\
\hline \multicolumn{4}{|c|}{ Portal lymphatic status ${ }^{a}$} \\
\hline No & 21 & 38 & 0.054 \\
\hline Yes & 3 & 0 & \\
\hline \multicolumn{4}{|l|}{ CXCR7 staining } \\
\hline Low & 5 & 23 & 0.002 \\
\hline High & 19 & 15 & \\
\hline
\end{tabular}

${ }^{\text {a}}$ Fisher's exact test.

Risk predictive role of $C X C R 7$ is related to OPN expression. Since OPN has been generally accepted as a factor closely related to the metastasis of $\mathrm{HCC}(17,18)$, we explored the potential association between the expression of CXCR7 and OPN. In this analysis, expression of OPN was divided into two groups: OPN ${ }^{\text {high }}$ and OPN ${ }^{\text {low }}$. Following stratification of category 1 based on cell differentiation (Edmondson grade 1/2) $(n=73)$, CXCR7 correlated well and positively with expression of OPN (Spearman's $\mathrm{Q}=0.240 ; \mathrm{P}=0.019)$. Furthermore, stratification analysis of category $2(n=62)$ confirmed the positive and high correlation between CXCR7 and OPN (Spearman's $\mathrm{Q}=0.453 ; \mathrm{P}<0.001)$. There was a significantly statistical difference between CXCR7 and OPN expression (Fisher's exact test, $\mathrm{P}=0.000)$. In addition, analyses based on immunostaining indicated that the distribution and strength of CXCR7 were consistently and closely associated with OPN in HCCs with well or moderate differentiation (Fig. 3A).

To confirm the potential regulatory role of CXCR7 in the expression of OPN, we detected the change in expression of OPN following downregulation of expression of CXCR7 in a highly metastatic HCC cell line. Both immunocytochemistry and RT-PCR found decreased expression of OPN following RNAi of CXCR7 (Fig. 3B).

\section{Discussion}

In the present study, we found that high expression of CXCR7 increased the risk of metastasis in HCC patients receiving hepatectomy, particularly when tumor cells were well and moderately differentiated. Stratification analysis based on Edmondson grade $1 / 2$ indicated that high expression of CXCR7 was a strong independent risk factor for metastasis. ROC analysis justified the predictive role of CXCR7. The results of category 2 further confirmed the results of category 1 . These findings indicated that CXCR7 was a valuable risk factor for predicting metastasis in patients with relatively well-differentiated HCC. Moreover, accumulation of chromosomal changes has been found to be associated with metastatic behavior, and that $\mathrm{LOH}$ on $16 \mathrm{q}$ is a useful prognostic indicator for metastasis after curative resection of HCC (19). In addition, HBsAg was shown to have a predictive role in extrahepatic metastasis after hepatic resection in patients with large hepatocellular carcinoma (20).

Generally, poor differentiation indicates a more invasive character of tumor cells. In the present study, however, more than half of the patients (73/116) had well or moderate differentiation, and lung metastasis occurred in 30 patients in this group. Although well differentiation is generally associated with a favorable prognosis, pathological factors such as CXCR7 were still used to stratify patients with respect to lung metastasis after resection in this study. Based on CXCR7 expression, we may recognize and distinguish the patients prone to lung metastasis from patients with relatively welldifferentiated HCC and intervene in advance. Therefore, this group of patients may acquire survival benefits.

Why does high expression of CXCR7 have a strong predictive role for lung metastasis in patients with relative well-differentiated HCC but not in patients with poor-differentiated HCC? One of the potential reasons is the heterogeneity of HCC $(21,22)$. Similarly to our study, previous research has shown that although early HCC is generally associated with a favorable prognosis, pathologic factors can still be used to stratify patients with respect to survival after resection (23). In addition, histological heterogeneity was found in small but established HCC, which was accompanied by increased proliferative activity and p53 protein overexpression. The expression of $\beta$-catenin protein also has heterogeneous distribution, even in the same histological grade region of the same tumor (24). Therefore, some HCC cells may acquire a new differentiation character such as high expression of CXCR7, 
which promotes the directional chemotaxis and metastasis of tumor cells to lung. On the other hand, apart from stimulating metastasis, research suggests other functions for CXCR7 such as its involvement in differentiation and development $(25,26)$. In poorly differentiated tumors, an embryonic stem cell-like gene expression signature suggests that CXCR7 is epigenetically controlled by SUZ12.

In the present study, we also found an association between CXCR7 and OPN in stratification analyses based on cell differentiation, which may be pertinent to the increased risk of extrahepatic metastasis. Furthermore, in this study, knockdown of CXCR7 in a highly metastatic HCC cell line induced the downregulation of OPN which was demonstrated by immunocytochemistry and mRNA detection. Our findings are similar to research on the role of CXCR7 in the early development of osteoarthritis, which found that overexpression of CXCR7 upregulated the expression of OPN 9-fold (27). Recent research has shown that CXCR7 participates in the non-classic pathway by activating mitogen-activated protein kinases through $\beta$-arrestin, (28) which may cross-talk with the Wnt signaling pathway. It is well known that OPN is the usual target of Wnt signaling in tumor progression $(29,30)$. The activated transmembrane CXCR7 may cross-talk with Wnt signaling through $\beta$-arrestin, and upregulate the expression of OPN, which further stimulates the metastatic ability of tumor cells. However, the exact mechanism deserves future investigation.

In conclusion, high expression of CXCR7 increases the risk of post-operative metastasis in patients with relatively well-differentiated HCC. The potential for early occurence of lung metastasis can be predicted in advance based on CXCR7 detection. This group of patients may acquire a survival benefit from the early detection and prevention of lung metastasis.

\section{Acknowledgements}

This study was supported by the State Key Basic Research Program Grant of the Ministry of Science and Technology, China (no. 2004CB518708), the Youth Fund from Zhongshan Hospital, and the Youth Backbone Fund from Fudan University (B-233).

\section{References}

1. Zhou XD: Recurrence and metastasis of hepatocellular carcinoma: progress and prospects. Hepatobiliary Pancreat Dis Int 1: 35-41, 2002.

2. Tang Z, Zhou X, Lin Z, et al: Surgical treatment of hepatocellular carcinoma and related basic research with special reference to recurrence and metastasis. Chin Med J 112: 887-891, 1999.

3. Balabanian K, Lagane B, Infantino S, et al: The chemokine SDF-1/CXCL12 binds to and signals through the orphan receptor RDC1 in T lymphocytes. J Biol Chem 280: 35760-35766, 2005.

4. Burns JM, Summers BC, Wang Y, et al: A novel chemokine receptor for SDF-1 and I-TAC involved in cell survival, cell adhesion, and tumor development. J Exp Med 203: 2201-2213, 2006.

5. Ben-Baruch A: Organ selectivity in metastasis: regulation by chemokines and their receptors. Clin Exp Metastasis 25: 345-356, 2008.

6. Wang J, Shiozawa Y, Wang Y, et al: The role of CXCR7/RDC1 as a chemokine receptor for CXCL12/SDF-1 in prostate cancer. J Biol Chem 283: 4283-4294, 2008.

7. Iwakiri S, Mino N, Takahashi T, et al: Higher expression of chemokine receptor CXCR7 is linked to early and metastatic recurrence in pathological stage I non small cell lung cancer. Cancer 115: 2580-2593, 2009.
8. Zheng K, Li HY, Su XL, et al: Chemokine receptor CXCR7 regulates the invasion, angiogenesis and tumor growth of human hepatocellular carcinoma cells. J Exp Clin Cancer Res 29: 31 , 2010.

9. Monnier J, Boissan M, L'Helgoualc'h A, et al: CXCR7 is up-regulated in human and murine hepatocellular carcinoma and is specifically expressed by endothelial cells. Eur J Cancer 48: 138-148, 2012.

10. Xue TC, Chen RX, Han D, et al: Down-regulation of CXCR7 inhibits the growth and lung metastasis of human hepatocellular carcinoma cells with highly metastatic potential. Exp Ther Med 3: 117-123, 2012.

11. Sun HC, Zhuang PY, Qin LX, et al: Incidence and prognostic values of lymph node metastasis in operable hepatocellular carcinoma and evaluation of routine complete lymphadenectomy. J Surg Oncol 96: 37-45, 2007.

12. Simon R, Mirlacher M and Sauter G: Tissue microarrays. Methods Mol Med 97: 377-389, 2004.

13. Lugli A, Spichtin H, Maurer R, et al: EphB2 expression across 138 human tumor types in a tissue microarray: high levels of expression in gastrointestinal cancers. Clin Cancer Res 11: 6450-6458, 2005.

14. Dalby B, Cates S, Harris A, et al: Advanced transfection with Lipofectamine 2000 reagent: primary neurons, siRNA, and high-throughput applications. Methods 33: 95-103, 2004.

15. Brooks SA: Basic immunocytochemistry for light microscopy. Methods Mol Biol 878: 1-30, 2012

16. Trevisani F, D'Intino PE, Morselli-Labate AM, et al: Serum alpha-fetoprotein for diagnosis of hepatocellular carcinoma in patients with chronic liver disease: influence of $\mathrm{HBsAg}$ and anti-HCV status. J Hepatol 34: 570-575, 2001.

17. Chen RX, Xia YH, Cui JF, Xue TC and Ye SL: Osteopontin, a single marker for predicting the prognosis of patients with tumornode-metastasis stage I hepatocellular carcinoma after surgical resection. J Gastroenterol Hepatol 25: 1435-1442, 2010.

18. Pan HW, Ou YH, Peng SY, et al: Overexpression of osteopontin is associated with intrahepatic metastasis, early recurrence, and poorer prognosis of surgically resected hepatocellular carcinoma. Cancer 98: 119-127, 2003.

19. Nishida N, Fukuda Y, Komeda T, et al: Prognostic impact of multiple allelic losses on metastatic recurrence in hepatocellular carcinoma after curative resection. Oncology 62: 141-148, 2002.

20. Sasaki A, Kai S, Endo Y, et al: Hepatitis B virus infection predicts extrahepatic metastasis after hepatic resection in patients with large hepatocellular carcinoma. Ann Surg Oncol 14: 3181-3187, 2007.

21. Emile JF, Lemoine A, Azoulay D, Debuire B, Bismuth H and Reynes M: Histological, genomic and clinical heterogeneity of clear cell hepatocellular carcinoma. Histopathology 38: 225-231, 2001.

22. Unsal H, Yakicier C, Marcais C, et al: Genetic heterogeneity of hepatocellular carcinoma. Proc Natl Acad Sci USA 91: 822-826, 1994.

23. Nathan H, Schulick RD, Choti MA and Pawlik TM: Predictors of survival after resection of early hepatocellular carcinoma. Ann Surg 249: 799-805, 2009.

24. An FQ, Matsuda M, Fujii H, et al: Tumor heterogeneity in small hepatocellular carcinoma: analysis of tumor cell proliferation, expression and mutation of p53 and $\beta$-catenin. Int $\mathbf{J}$ Cancer 93: 468-474, 2001.

25. Ben-Porath I, Thomson MW, Carey VJ, et al: An embryonic stem cell-like gene expression signature in poorly differentiated aggressive human tumors. Nat Genet 40: 499-507, 2008.

26. Lee TI, Jenner RG, Boyer LA, et al: Control of developmental regulators by Polycomb in human embryonic stem cells. Cell 125: 301-313, 2006.

27. Jones SW, Brockbank SM, Mobbs ML, et al: The orphan G-protein coupled receptor RDC1: evidence for a role in chondrocyte hypertrophy and articular cartilage matrix turnover. Osteoarthritis Cartilage 14: 597-608, 2006.

28. Rajagopal S, Kim J, Ahn S, et al: Beta-arrestin- but not G protein-mediated signaling by the'decoy' receptor CXCR7. Proc Natl Acad Sci USA 107: 628-632, 2010.

29. Ravindranath A, Yuen HF, Chan KK, et al: Wnt- $\beta$-catenin-Tcf-4 signalling-modulated invasiveness is dependent on osteopontin expression in breast cancer. Br J Cancer 105: 542-551, 2011.

30. Rohde F, Rimkus C, Friederichs J, et al: Expression of osteopontin, a target gene of de-regulated Wnt signaling, predicts survival in colon cancer. Int J Cancer 121: 1717-1723, 2007. 